\title{
Cardiac and inflammatory biomarkers do not correlate with volume of heart or lung receiving radiation
}

\author{
Angera H Kuo ${ }^{1,2}$, Marek Ancukiewicz ${ }^{1}$, Kevin R Kozak ${ }^{1,3}$, Torunn I Yock ${ }^{1}$ and Timothy P Padera ${ }^{\text {1* }}$
}

\begin{abstract}
Background: Thoracic and cardiac irradiation increases the risk of pulmonary and cardiovascular disease. In addition, radiation, often in combination with chemotherapy, can cause treatment-related pneumonitis. Previously, we showed that the common marker for cardiac damage, troponin T, was not elevated by chemoradiation [Lung Cancer 62:351-355, 2008]. In this study, we explore whether dose-volume metrics and biomarkers for cardiac damage, inflammation or angiogenesis could identify patients receiving thoracic radiation who would later have cardiac or pulmonary complications.

Findings: To this end, we quantified cardiac biomarkers including c-reactive protein (cRP) as well as a panel of angiogenic and inflammatory molecules in thirty patients who received radiation therapy to the thorax with or without concurrent chemotherapy between May 2006 and May 2007. Serum was collected at baseline, 2 weeks into radiation treatment and at the completion of radiation therapy. Heart and lung dosimetric parameters and clinical risk factors were also examined, along with the monitoring of adverse pulmonary and cardiac events during follow-up. Contrary to our hypothesis, there was no correlation between serum biomarker levels and cardiac radiation dose. Similarly there was little association between lung dose-volume metrics and inflammatory or angiogenic biomarkers. Furthermore, there was no correlation with serum biomarkers and adverse pulmonary or cardiovascular events.
\end{abstract}

Conclusion: Based on these data, acute elevations in serum biomarkers of cardiac damage, inflammation or angiogenesis should not be attributed to thoracic (chemo)radiation and elevations in such biomarkers of tissue damage should be further evaluated.

Keywords: Radiation, Lung cancer, Cardiotoxicity, Radiation induced pneumonitis, Biomarkers, C-reactive protein

\section{Findings}

\section{Background and motivation}

The use of radiation to treat thoracic cancers can cause treatment-related pneumonitis [1,2]. Similarly, cardiac mortality increases in patients treated with radiation to the chest [3-5]. To date, there are no validated biomarkers to predict which patients will develop these side effects. Because these patient populations tend to have extensive co-morbidities, as well as a generally poor overall prognosis, the harmful effects of thoracic irradiation do not

\footnotetext{
* Correspondence: tpadera@steele.mgh.harvard.edu

'Department of Radiation Oncology, MGH Cancer Center, Massachusetts General Hospital and Harvard Medical School, Cox-737, 100 Blossom Street, Boston, MA 02114, USA

Full list of author information is available at the end of the article
}

necessarily manifest clinically in many patients. However, as survival continues to improve with better systemic therapies and more effective radiation dose delivery, greater focus is needed on the toxicities of thoracic radiotherapy.

Certain markers of inflammation (i.e. Interleukin-6) rise in patients that go on to develop pneumonitis [6,7], however no study has evaluated a large panel of inflammatory markers in individual patients. Furthermore, cardiac disease risk factors and symptoms overlap with those of thoracic malignancies, which might mask early signs of cardiac disease. Thus understanding how known biomarkers of cardiac damage change during thoracic radiotherapy might improve early detection of cardiac compromise and may allow real-time modification of radiation treatment to prevent further toxicity. Robust 
predictive biomarkers of radiation induced cardiac or pulmonary pathologies would permit identification of patients that require intense monitoring after radiation treatment. These patients may potentially benefit from earlier intervention to limit morbidity from radiation induced pathologies.

\section{Materials and methods}

Serum samples were used from a cohort of patients previously published in [8] and summarized in Table 1. Briefly, between May 2006 and May 2007, blood was collected from 30 patients older than 18 years undergoing threedimensional conformal or intensity modulated radiation therapy for a thoracic malignancy at Massachusetts General Hospital. Patients were excluded if they had a history of renal failure, hematocrit of less than $25 \%$ or previous thoracic radiotherapy. The study was approved by the Human Research Committee of Partners Healthcare and written informed consent was obtained from all patients. The average serum storage time at $-80^{\circ} \mathrm{C}$ was approximately 30 months, which should maintain stability of the analytes including cRP [9]. Each patient gave three samples: the first obtained 0-8 days prior to radiation therapy (Day 0), the second

Table 1 Patient characteristics $(\mathbf{N}=30)$

\begin{tabular}{|c|c|c|}
\hline Characteristic & $\mathrm{N}$ & $\%$ \\
\hline Male & 17 & $57 \%$ \\
\hline Female & 13 & $43 \%$ \\
\hline \multicolumn{3}{|l|}{ Diagnosis } \\
\hline Non-small cell lung cancer & 18 & $60 \%$ \\
\hline Small cell lung cancer & 4 & $13 \%$ \\
\hline Esophageal cancer & 5 & $17 \%$ \\
\hline Gastroesophageal junction cancer & 2 & $7 \%$ \\
\hline Thymic carcinoma & 1 & $3 \%$ \\
\hline \multicolumn{3}{|l|}{ Stage } \\
\hline । & 1 & $3 \%$ \\
\hline$\|$ & 6 & $20 \%$ \\
\hline III & 13 & $43 \%$ \\
\hline IV & 5 & $17 \%$ \\
\hline Limited small cell lung cancer & 4 & $13 \%$ \\
\hline Recurrent & 1 & $3 \%$ \\
\hline \multicolumn{3}{|l|}{ Prior resection } \\
\hline Yes & 7 & $23 \%$ \\
\hline No & 23 & $77 \%$ \\
\hline \multicolumn{3}{|l|}{ Induction chemotherapy } \\
\hline Yes & 7 & $23 \%$ \\
\hline No & 23 & $77 \%$ \\
\hline \multicolumn{3}{|l|}{ Concurrent chemotherapy } \\
\hline Yes & 24 & $80 \%$ \\
\hline No & 6 & $20 \%$ \\
\hline
\end{tabular}

following 8-10 fractions of radiation therapy (Day 10) and the third at the end of radiation therapy (Day 42). Cardiac and pulmonary dose-volume metrics were collected from approved treatment plans by a radiation oncologist and entire heart and lungs were contoured on radiation planning $\mathrm{CT}$ images. These data are summarized in Table 2. Adverse cardiac and pulmonary events were diagnosed clinically and recorded.

Serum samples were analyzed using multiplex ELISA from Mesoscale Discovery (Rockville, MD) and read on a Mesocsale Discovery multiplex plate reader (Sector Imager 2400). Each sample was run in duplicate on each of 3 different multiplex ELISA arrays to include the following 18 analytes: c-reactive protein (cRP), serum amyloid A (SAA), vascular cell adhesion molecule-1 (VCAM-1) and intracellular adhesion molecule-1 (ICAM-1) (Vascular Injury II kit; Mesoscale Discovery); interleukin (IL)-1 $\beta$, IL-2, IL-4, IL-5, IL-6, IL-8, IL-10, IL-12, granulocytemacrophage colony-stimulating factor (GM-CSF) and tumor necrosis factor- $\alpha$ (TNF- $\alpha$ ) (Human Demonstration 10-Plex Tissue Culture Kit; Mesoscale Discovery); basic fibroblast growth factor (bFGF), placental growth factor (PlGF), vascular endothelial growth factor (VEGF) and soluble VEGF receptor-1 (sVEGFR-1) (Human Growth Factor I Kit; Mesoscale Discovery).

\section{Statistics}

Principal component analysis (PCA) was performed to reduce the number of variables characterizing dosimetry in order to relate dosimetry to clinical events. Dosimetry is described by the mean dose to the organ (i.e. heart or lungs), the maximum dose to the organ and the percent of the organ volume that received a dose of at least 5, 10, 15, 20, 30, 40, 50, 60 and 70 Gy. The first principal component for 11 variables describing the dose to the heart (Hmax, Hmean, HV5, HV10, HV15, HV20, HV30, HV40, HV50, HV60, HV70) accounted for $61 \%$ of the variance and inversely correlated with Hmean, HV5, HV10, HV15, HV20 and HV30. The first principal component for 11 variables describing the dose to the lungs (Lmax, Lmean, LV5, LV10, LV15, LV20, LV30, LV40, LV50, LV60, LV70) accounted for $52 \%$ of the variance and inversely correlated with Lmean, LV5, LV10, LV15, LV20 and LV30. The correlation of dosimetric parameters with bioanalyte protein levels, as well as the correlation between cRP and SAA, was assessed using Kendall's test with Benjamini-Hochberg adjustments for multiple comparisons [10]. Based on our sample, we could detect Kendall's tau $=0.34$ with a power of $80 \%$ as statistically significant with $\mathrm{p}<0.05$. Follow-up time was calculated using the "reverse Kaplan-Meier" Method described by Schemper and Smith [11]. To determine whether SAA values in individual patients increased or decreased after 10 days of radiation therapy, we used two criteria, as the baseline 
Table 2 Radiation dosimetry for the heart and lungs $(n=30)$

\begin{tabular}{|c|c|c|c|c|}
\hline \multirow[b]{2}{*}{ Parameter } & \multicolumn{2}{|l|}{ Cardiac } & \multicolumn{2}{|l|}{ Pulmonary } \\
\hline & Median (range) & Mean \pm SEM & Median (range) & Mean \pm SEM \\
\hline Mean organ dose (Gy) & $12.7(0.6-41.0)$ & $13.4 \pm 1.9$ & $14.0(3.3-34.3)$ & $14.1 \pm 1.1$ \\
\hline Maximum organ dose (Gy) & $50.3(1.6-73.4)$ & $47.0 \pm 3.7$ & $57.9(42.7-80.5)$ & $61.3 \pm 1.9$ \\
\hline V5Gy (\%) & $63.4(0-100)$ & $55.7 \pm 6.8$ & $61.3(10.0-92.3)$ & $58.1 \pm 4.2$ \\
\hline V10Gy (\%) & $44.8(0-100)$ & $42.1 \pm 5.9$ & $43.5(7.9-87.1)$ & $42.7 \pm 3.4$ \\
\hline V15Gy (\%) & $31.5(0-100)$ & $33.2 \pm 5.3$ & $34.5(7.1-82.2)$ & $34.0 \pm 2.8$ \\
\hline V20Gy (\%) & $22.1(0-100)$ & $27.3 \pm 4.9$ & $26.6(5.1-78.0)$ & $27.2 \pm 2.6$ \\
\hline V30Gy (\%) & $11.9(0-87.3)$ & $16.9 \pm 3.7$ & $14.3(2.4-65.3)$ & $16.9 \pm 2.2$ \\
\hline V40Gy (\%) & $4.3(0-65.0)$ & $9.4 \pm 2.7$ & $8.3(0.3-42.2)$ & $10.4 \pm 1.7$ \\
\hline V50Gy (\%) & $0(0-15)$ & $2.0 \pm 0.6$ & $3.0(0-20.0)$ & $4.5 \pm 0.9$ \\
\hline V60Gy (\%) & $0(0-3.2)$ & $0.3 \pm 0.1$ & $0(0-8.1)$ & $1.8 \pm 0.5$ \\
\hline V70Gy (\%) & $0(0-0.2)$ & 0 & $0(0-2.6)$ & $0.2 \pm 0.1$ \\
\hline
\end{tabular}

VXGy (\%) represents the percent of the total organ volume receiving a dose of at least X Gy.

SAA levels in this patient population varied over 2 orders of magnitude. A rise in SAA was determined by a $50 \%$ increase in SAA from Day 0 to Day 10 as well as at least a $10 \mathrm{ng} / \mathrm{ml}$ increase in SAA. A decrease in SAA was determined by a $50 \%$ decrease in SAA from Day 0 to Day 10 as well as at least a $10 \mathrm{ng} / \mathrm{ml}$ decrease in SAA. The same criteria were used for cRP (using $10 \mu \mathrm{g} / \mathrm{ml}$ as the absolute cut-off).

\section{Results}

\section{Patient follow-up}

Extending the average follow-up time to 3.4 years of the patient cohort previously published [8], four out of the 30 patients developed pneumonitis after radiation and 12 of the 30 patients had cardiovascular events (myocardial infarction, stroke, deep vein thrombosis, atrial fibrillation, pulmonary embolism). Complications were recorded solely based on a clinical diagnosis of the event.

\section{Serum amyloid A (SAA) is elevated during radiation therapy}

Of the 18 analytes measured in patients receiving thoracic radiation, only SAA - a protein associated with inflammatory stimuli in a variety of settings $[12,13]$ —showed a statistically significant change during the course of therapy. SAA was elevated at Day 10 when taking the average of all 30 patients (Figure 1A). However, individual patients responded differently, with only 14 of the 30 patients seeing increased levels of SAA at Day 10, while 8 patients had decreased levels and 8 patients showed no difference (Figure 1B). The first principal component of cardiac dosimetry was related to the average SAA levels at Days 10 and 42. Similarly the mean radiation dose to the heart correlated to the average SAA levels at the same time points. Additionally, there was a significant correlation between the percent volume of the heart receiving 5 Gy and SAA levels at Days 10 and 42. However, this correlation with SAA levels was not found when the percent volumes of the heart receiving 30 or 50 Gy were tested.

Our data also showed a very weak association with levels of cRP and cardiac dosimetry. 10 of the 30 patients showed increases in cRP at Day 10, where only 4 patients showed a decrease (Figures 1C,D). However, the magnitude of the increase in cRP was not large enough to detect a statistically significant difference in this population. SAA and cRP circulating protein levels positively correlated for each individual time point (Kendall's $\mathbf{\tau}=0.71,0.81$ and 0.65 at Day 0, 10 and 42, respectively; p $<0.05$; Figure 2), suggesting that they are mirroring the same underlying biology. Furthermore, changes from Day 0 in SAA and cRP circulating protein levels also positively correlate (Kendall's $\mathbf{\tau}=0.64$ and 0.63 for changes from day 0 to day 10 and 42, respectively; $\mathrm{p}<0.05$ ), suggesting that these proteins rise and fall together and share a common response mechanism. However from these data, SAA is a more sensitive marker of radiation dose to the heart.

Even though there was an increase in SAA levels at Day 10 , the levels trended back to baseline by the end of therapy. Importantly, there was no correlation between patients with a rise in SAA and patients that eventually had cardiovascular (or pulmonary) events. Thus the rise in SAA was not predictive of a worse cardiovascular outcome in this patient population. The other 16 analytes measured did not show any correlation to cardiac dosimetry or the incidence of cardiac events (data not shown).

\section{Inflammatory biomarkers are not correlated with pneumonitis}

It is known that the dose of radiation to the lungs is correlated with pneumonitis [1]. The data from our patient sample are consistent with this finding, as the 

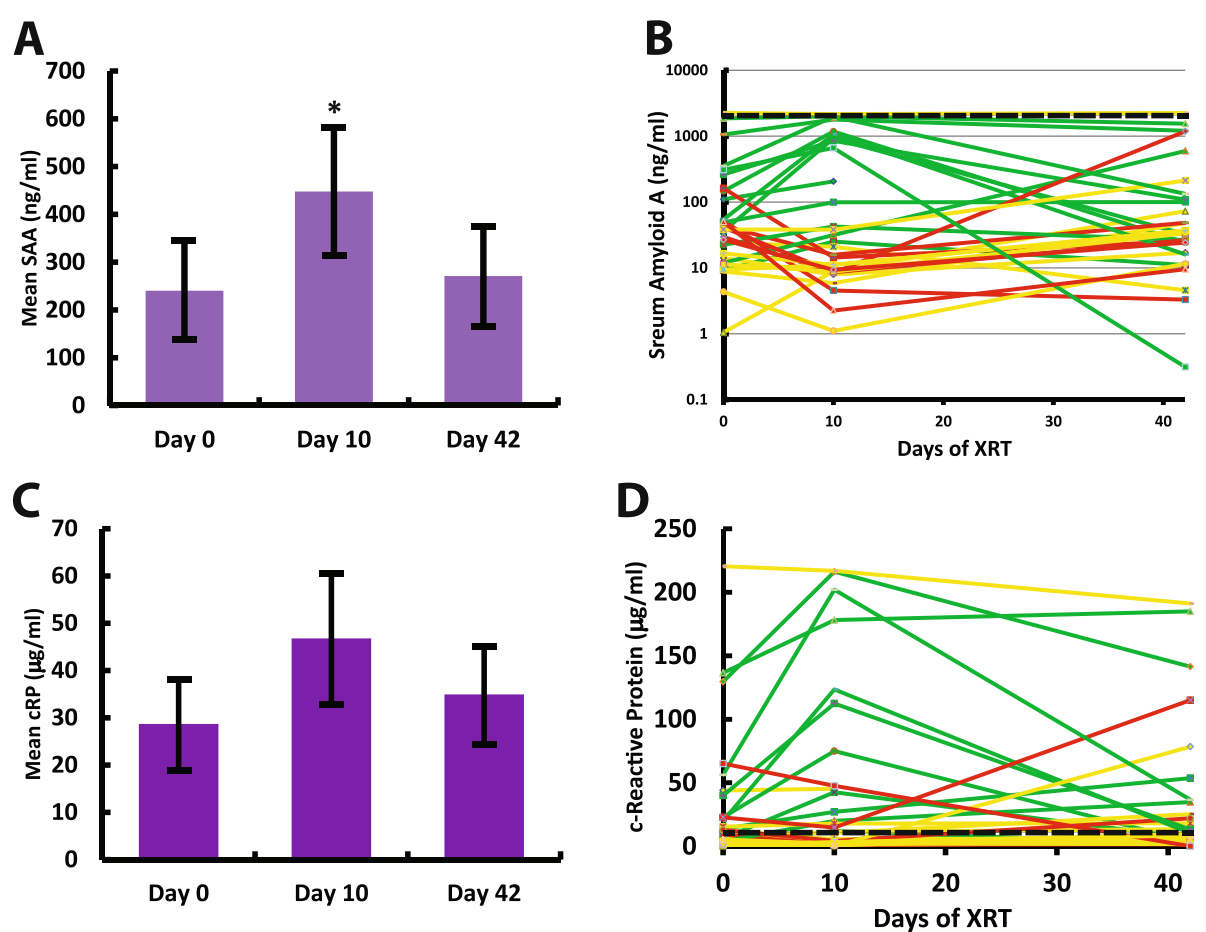

Figure 1 Serum amyloid A (SAA) rises in select patients during thoracic radiation. A) Average SAA rose during thoracic radiation and returned to pre-treatment levels after radiotherapy was complete. B) Individual patients showed heterogeneity in the response of SAA during thoracic radiation. Green lines show patients with increased levels of SAA after 10 days of radiotherapy. Red lines show patients with decreased levels of SAA after 10 days of radiotherapy. Yellow lines show patients with no change in SAA after 10 days of radiotherapy. Dashed line shows upper limit of normal values for SAA (2000 ng/ml). Data plotted on log scale to highlight the three orders of magnitude spread in the range of values. C) c-Reactive Protein (CRP) showed an elevated trend during radiation but similarly returned to pre-treatment levels after radiotherapy. D) Individual patients showed heterogeneity in the response of CRP during thoracic radiation. Green lines show patients with increased levels of cRP after 10 days of radiotherapy. Red lines show patients with decreased levels of cRP after 10 days of radiotherapy. Yellow lines show patients with no change in CRP after 10 days of radiotherapy. Dashed line shows upper limit of normal values for cRP $(10 \mu \mathrm{g} / \mathrm{ml})$. Data in (a) and (c) are plotted as mean \pm SEM. ${ }^{*} p<0.05$.

pneumonitis incidence correlated with the first principal component of pulmonary dosimetry $(\mathrm{p}<0.05)$, with higher doses corresponding to the incidence of pneumonitis. However, none of the 18 serum biomarkers correlated with lung dose-volume metrics (data not shown). Furthermore, there was no correlation between these biomarkers and the incidence of pneumonitis. Thus, based on this patient sample, these biomarkers are not robust in predicting pneumonitis in patients receiving thoracic radiation.

\section{Conclusions}

Although our patient sample is limited, no strong candidate serum biomarker emerged, which is a necessary condition for patient stratification. Furthermore, individual patient responses varied greatly (Figures $1 \mathrm{~B}, \mathrm{D}$ ), making a prediction in any particular patient even more challenging. Nevertheless, small rises in cRP and SAA can be expected when the heart receives radiation in the course of thoracic radiotherapy, suggesting that they are not highly specific biomarkers if a cardiac event is suspected in this setting. These patients need alternative tests if cardiac events are

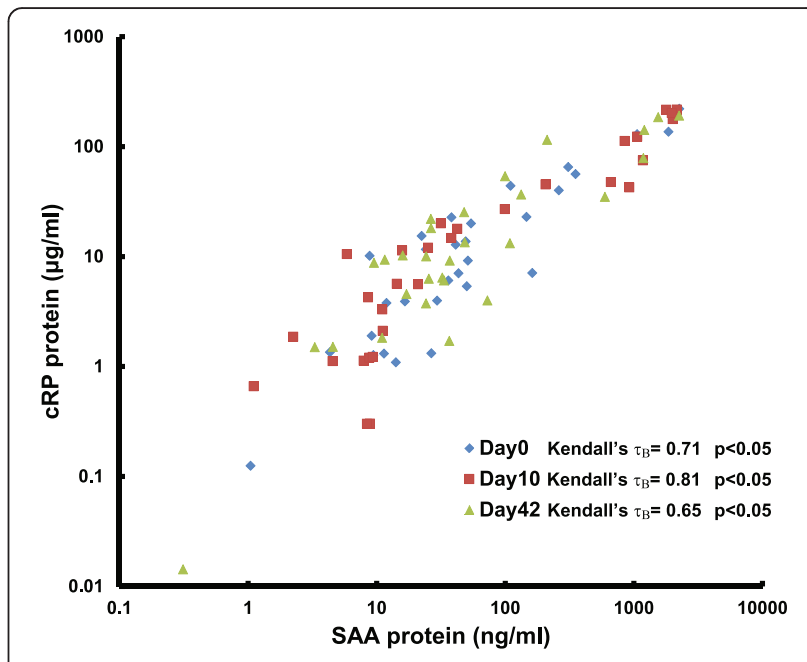

Figure 2 SAA and CRP are strongly correlated. The correlation of SAA and CRP is independent of the timing the sample was taken during the course of thoracic radiation therapy. 
suspected during therapy. Surprisingly, lung radiation did not elevate the biomarkers we measured. However, even in this small study, the volume of lung and dose received correlated to the development of pneumonitis, consistent with the literature on this topic [2]. Thus, a predictive serum biomarker for pneumonitis in the acute phase remains elusive [7].

\section{Abbreviations}

CRP: c-reactive protein; IL: Interleukin; PCA: Principal component analysis; SAA: Serum amyloid A.

\section{Competing interests}

The authors declare that they have no competing interests with this work. KRK is Chief Medical Officer, Co-D Therapeutics, Inc. and Cellectar Biosciences, Inc., which have no clear relationship to the presented work.

\section{Authors' contributions}

AHK performed experiments and analyzed data. MA performed the statistical analysis. KRK obtained IRB approval, collected patient samples and measured dose-volume metrics. TIY obtained IRB approval and collected clinical data. TPP analyzed data and drafted the manuscript. All authors read and approved the final manuscript.

\section{Acknowledgements}

We thank Anna Khachatryan for her outstanding technical support. This work was supported by an Ira J. Spiro Translational Research Award (TIY, TPP), NIH R00CA137167 (TPP) and NIH DP2OD008780 (TPP)

\section{Author details}

${ }^{1}$ Department of Radiation Oncology, MGH Cancer Center, Massachusetts General Hospital and Harvard Medical School, Cox-737, 100 Blossom Street, Boston, MA 02114, USA. ${ }^{2}$ Current Affiliation: Institute for Stem Cell Biology and Regenerative Medicine, Stanford University School of Medicine, Stanford, CA, USA. ${ }^{3}$ Current Affiliation: Mercy Regional Cancer Center, Janesville, WI, USA

Received: 28 July 2014 Accepted: 27 November 2014

Published online: 09 January 2015

\section{References}

1. Graham MV, Purdy JA, Emami B, Harms W, Bosch W, Lockett MA, et al. Clinical dose-volume histogram analysis for pneumonitis after 3D treatment for non-small cell lung cancer (NSCLC). Int J Radiat Oncol Biol Phys. 1999:45:323-9.

2. Marks LB, Bentzen SM, Deasy JO, Kong FM, Bradley JD, Vogelius IS, et al. Radiation dose-volume effects in the lung. Int J Radiat Oncol Biol Phys. 2010;76:570-6.

3. Postoperative radiotherapy in non-small-cell lung cancer: systematic review and meta-analysis of individual patient data from nine randomised controlled trials. PORT Meta-analysis Trialists Group. Lancet 1998, 352:257-263.

4. Dautzenberg B, Arriagada R, Chammard AB, Jarema A, Mezzetti M, Mattson $\mathrm{K}$, et al. A controlled study of postoperative radiotherapy for patients with completely resected nonsmall cell lung carcinoma. Groupe d'Etude et de Traitement des Cancers Bronchiques. Cancer. 1999;86:265-73.

5. Mukherjee S, Aston D, Minett M, Brewster AE, Crosby TD. The significance of cardiac doses received during chemoradiation of oesophageal and gastro-oesophageal junctional cancers. Clin Oncol. 2003;15:115-20.

6. Chen Y, Williams J, Ding I, Hernady E, Liu W, Smudzin T, et al. Radiation pneumonitis and early circulatory cytokine markers. Semin Radiat Oncol. 2002;12:26-33

7. Stenmark MH, Cai XW, Shedden K, Hayman JA, Yuan S, Ritter T, et al. Combining physical and biologic parameters to predict radiation-induced lung toxicity in patients with non-small-cell lung cancer treated with definitive radiation therapy. Int J Radiat Oncol Biol Phys. 2012;84:e217-22.

8. Kozak KR, Hong TS, Sluss PM, Lewandrowski EL, Aleryani SL, Macdonald SM, et al. Cardiac blood biomarkers in patients receiving thoracic (chemo) radiation. Lung Cancer. 2008:62:351-5.
9. Brindle E, Fujita M, Shofer J, O'Connor KA. Serum, plasma, and dried blood spot high-sensitivity C-reactive protein enzyme immunoassay for population research. J Immunol Methods. 2010;362:112-20.

10. Benjamini Y, Hochberg Y. A practical and powerful approach to multiple testing. J R Stat Soc B. 1995;57:289-300.

11. Schemper $M$, Smith TL. A note on quantifying follow-up in studies of failure time. Control Clin Trials. 1996;17:343-6.

12. Malle $E$, De Beer FC. Human serum amyloid A (SAA) protein: a prominent acute-phase reactant for clinical practice. Eur J Clin Invest. 1996;26:427-35.

13. Malle E, Sodin-Semrl S, Kovacevic A. Serum amyloid A: an acute-phase protein involved in tumour pathogenesis. Cell Mol Life Sci. 2009;66:9-26.

\section{Submit your next manuscript to BioMed Central and take full advantage of:}

- Convenient online submission

- Thorough peer review

- No space constraints or color figure charges

- Immediate publication on acceptance

- Inclusion in PubMed, CAS, Scopus and Google Scholar

- Research which is freely available for redistribution 